\title{
The nascent field of AllergoOncology
}

\author{
Erika Jensen-Jarolim • Graham Pawelec
}

Received: 13 May 2012/ Accepted: 29 June 2012/Published online: 22 August 2012

(C) Springer-Verlag 2012

\begin{abstract}
Many intriguing relationships between allergy and cancer continue to pose important unanswered questions. Is there a correlation between IgE and atopy, and cancer? Which are the most important IgE effector cells and do they inhibit or promote tumor growth? Will IgEmediated antigen uptake and cross-presentation by DCs activate CTLs or Tregs? What animal models are useful in this context and should we design specific anti-tumor immunotherapies, active or passive, based on IgE? Recombinant IgE has already been generated against the tumor-associated antigens folate-binding receptor, HER-2 and EGFR-how to exclude the risk of anaphylaxis in passive immunotherapy with IgE? How can we deal with hypersensitivity to antineoplastic drugs? What is the role of chemotherapeutics and biologicals in the modulation of IgE responses and the impact of the aberrant expression of mutated enzyme AID in cancer tissue? In an effort to survey the state-of-the-art in this area and to answer some of these questions, we invited leaders in the field to
\end{abstract}

This Editorial forms an introduction to the Symposium-in-Writing: "AllergoOncology: The Role of Th2 responses in cancer". We thank Prof. Erika Jensen-Jarolim for compiling and co-editing this series of papers and all authors and peer-reviewers for their contributions.

E. Jensen-Jarolim ( $\square)$

Comparative Medicine, Messerli Research Institute,

University of Veterinary Medicine Vienna,

Medical University Vienna, University Vienna,

c/o Inst. of Pathophysiology and Allergy Research,

Währinger G. 18-20, 1090 Vienna, Austria

e-mail: erika.jensen-jarolim@meduniwien.ac.at

G. Pawelec

Center for Medical Research (ZMF), University of Tübingen

Medical School, Waldhörnlestraße 22,

72072 Tübingen, Germany participate in a "Symposium-in-Writing". This represents a collection of peer-reviewed papers covering each group's specialist area of expertise. In this way, we aim to target the most important topics in these areas and to provide a comprehensive view of the state-of-the-art in the emerging multi-disciplinary field of "AllergoOncology".

Keywords AllergoOncology · Symposium-in-Writing · IgE $\cdot$ Cancer $\cdot$ Immunotherapy

The emerging interdisciplinary field of AllergoOncology represents a multifaceted attempt to determine the interrelations of cancer and the Th2 branch of the immune system, which has recently greatly expanded from clinical observations to an understanding of the molecular mechanism leading to improved targeted therapies. In the first paper, Turner [1] describes epidemiological investigations confirming potential inverse associations between a history of allergies and the risk of malignancies. This shows that significant inverse associations are observed in pancreatic cancer, glioma and childhood leukemia, but that more studies are needed in other cancers. Numerous confounding factors may be involved here, ranging from the way in which allergies are diagnosed (by a physician, by questionnaire, standardized or self-reported?), gene polymorphisms and regulatory proteins and the type of cancer. The methods employed bias the resulting picture and make it challenging to compare different studies. Regarding mechanisms, the overall working hypothesis is that allergens may crosslink IgE antibodies fixed to effector cells by means of allergen-associated molecular patterns (AAMPs), analogous to tumor antigens by tumor-associated molecular patterns (TAMPs). The resulting immune response is on the one hand inappropriate hypersensitivity toward 
innocuous allergens, but on the other the activation of anticancer inflammation, the overall result of which will depend on the type of effector cells involved. For instance, typical IgE-bearing cells such as mast cells accumulate in the microenvironment of both benign and malignant tumors. Because they are a source of pro-angiogenic factors, mast cell accumulation is often interpreted as a sign of poor prognosis, which is corroborated by clinical data. In contrast, Dalton et al. [2] provide evidence that loss of c-kit associated with the absence of mast cells in a mouse model results in a significant enhancement of intra-tumoral CTL and Th1 lymphocyte responses. The authors propose that intra-tumoral mast cells induce tolerogenic DCs which actively suppress T-cell responses. However, this situation may completely change in the presence of IgE. Platzer et al. [3] analyze whether an IgE-driven response might be associated with CTL activity. In their view, $\operatorname{IgE}$ (as is well known for $\mathrm{IgG}$ ) may be used for focusing exogenous antigens (such as tumor antigens) to DCs, resulting in more effective antigen presentation, cross-presentation and activation of anti-tumor $\mathrm{T}$ lymphocytes, but also possibly cross-activation of Tregs. This unconventional pathway has been ignored so far and should be further investigated so that it can be exploited to the benefit of the patient.

Like mast cells, the eosinophilic granulocyte is also a classic IgE effector cell. Tumor-associated tissue eosinophilia (TATE) has been associated with either a positive or a negative prognosis in different clinical studies and cancer types. Gatault et al. [4] point out that eosinophils have the potential to act via innate and adaptive immune mechanisms. Even more than the mast cell, the eosinophil may exert anti-tumor activity and is indeed most often found in a degranulated state in the tissue around cancer cells. These authors note that close contact between the eosinophil and the target cell results in apoptosis and necrosis of the latter. It is likely that an IL-5-rich environment primes the eosinophils for anti-tumor activity. Whether $\operatorname{IgE}$ is in fact involved in this interaction still requires further investigation, but this is hampered by a lack of anti-tumor antibodies of the IgE isotype. However, the eosinophil is perfectly equipped for such functions by the expression of both lowand high-affinity IgE receptors, at least in humans. This leads us to a consideration of the apparent limitations of animal models for AllergoOncology investigations. Daniels et al. [5] review the presently available mouse models of potential use in this context. For proof of concept (PoC) studies of anti-tumor vaccines, for example, using peptides or mimotopes, wild-type immunocompetent mouse models are needed. However, as mice lack the expression of the high-affinity receptor for IgE, FceRI, on eosinophils and DCs, this approach is limited and results are most likely not directly translatable to humans. The same applies to PoC studies of passive immunotherapy using murine $\operatorname{IgE}$ antibodies where the different distribution pattern of Fc\&RI receptors in humans and rodents must be taken into account. When testing humanized or chimeric recombinant IgE antibodies in human xenograft models, passively applied human PBMCs were previously successfully used as effector cells and support the concept that $\operatorname{IgE}$ is at least as cytotoxic as IgG, if not more so. There are some possibilities to adapt existing models such as using human FceRI alpha chain transgenic mice, which have the same pattern of expression of $\mathrm{Fc}$ receptors as humans.

Considering the impressive number of $\mathrm{IgG}$ antibodies now in clinical use or in the preclinical pipeline, the use of IgE anti-cancer antibodies is still in the early stage. Karagiannis et al. [6] were the first to compare $\operatorname{IgE}$ and $\operatorname{IgG}$ antibodies (anti-folate receptor) in a SCID xenotransplant mouse model, and recently also developed an anti-HER-2 antibody of the IgE isotype. Complementary to the in vivo studies, new in vitro systems were also developed to analyze isotype-specific killing functions of IgE. These assays indicate that monocytes are also important players in the context of $\operatorname{IgE}$, and that $\operatorname{IgE}$ is in fact better at inducing cytotoxicity (ADCC) than phagocytosis (ADCP) in cancer. Such assays were also adapted and applied by Spillner et al. [7] who discuss EGFR, a well-known target in clinical oncology, as a novel and interesting target for an $\mathrm{Al}-$ lergoOncology approach. Both papers [6, 7], however, emphasize that safety studies still need to be undertaken before using anti-tumor $\operatorname{IgE}$ in patients. Anaphylaxis is particularly strongly associated in our minds with the $\operatorname{IgE}$ antibody class. However, this ignores the obvious circumstance that we all express IgE without consequences as long as polyvalent allergen is absent. It has further been demonstrated that cancer patients also harbor natural antitumor IgE without any episodes of systemic reactions. This may be due to a natural safety measure preventing $\operatorname{IgE}$ crosslinking in the circulation: cancer antigens such as HER-2 or EGFR seem to be shed from the tumor cell only in monovalent form.

On the other hand, the systemic side effects of conventional therapy are a well-known oncological problem. In most infusion therapies with chemotherapeutics or biologicals, pre- or co-medication with cortisone or anti-histamines has to be carried out prophylactically, but systemic side effects still occur and may also result in hypersensitivity to antineoplastic agents or biologicals. This problem is presented by Castells et al. [8] as a common issue limiting clinical application because of the fear of possibly lethal systemic reactions. This often forces the clinical oncologist to change from first-line to suboptimal anticancer therapy. Careful protocols have therefore been elaborated to induce transient tolerance to the drug by rapid desensitization. There is also the possibility that chemotherapeutics may interfere with IgE synthesis, although the 
IgE parameters (total or specific) in chemotherapy are generally poorly established and may be regimen dependent. Bluth [9] describes the situation with temozolomide, which, like other alkylating drugs, potentiates IgE production. This may be due to elimination of suppressor or regulatory $\mathrm{T}$ cells during antineoplastic therapy. Interference with hormone status by some drug analogs, such as tamoxifen or octreotide, has also been shown to lead to the elevation of $\operatorname{IgE}$ and $\operatorname{IgG}$ titers. Clearly, these areas need to be explored in the future in order to better understand the complexities of interference with Th2 immune status.

Finally, Mechtcheriakova et al. [10] propose that allergies and malignancies are even more tightly connected by a single enzyme as a "missing link", namely activationinduced cytidine deaminase (AID). This enzyme introduces point mutations into sites where sequence variability is prone to affect function. It is responsible for isotype switch recombination and somatic hypermutation in B lymphocytes, acting as a crucial factor for affinity maturation. AID and IgE production have been described in ectopic lymph follicles and hyperplastic epithelia, and this enzyme is strongly expressed in cancer cells. It induces mutations in oncogenes, driving immune escape and affecting the strength and duration of the oncogenic signal. AID in cancer may thus support aberrant IgE production at the tumor site with predicted tumoricidal effects, but simultaneously facilitate disease progression.

Within this Symposium-in-Writing, contributions from opinion leaders within the emerging field of "AllergoOncology" have been solicited, with the aim of expounding novel concepts in this interdisciplinary approach and providing a state-of-the-art overview.

Acknowledgments E. J.-J. is supported by the Austrian Science Fund project P 23398-B11. G.P. is supported by the European Commission [FP7 259679 "IDEAL"]; German Research Foundation [DFG-PA 361/14-1]; German Federal Ministry of Education and Research [BMBF 0315890F, "Gerontoshield"].

Conflict of interest The authors declare that they have no conflict of interest.

\section{References}

1. Turner MC (2011) Epidemiology: allergy history, IgE, and cancer. Cancer Immunol Immunother. Online First ${ }^{\mathrm{TM}} 20$ Dec 2011. doi: 10.1007/s00262-011-1180-6

2. Dalton DK, Noelle RJ (2012) The roles of mast cells in anticancer immunity. Cancer Immunol Immunother. Online First ${ }^{\mathrm{TM}} 18 \mathrm{Apr}$ 2012. doi: $10.1007 / \mathrm{s} 00262-012-1246-0$

3. Platzer B, Dehlink E, Turley SJ, Fiebiger E (2011) How to connect an IgE-driven response with CTL activity? Cancer Immunol Immunother. Online First ${ }^{\mathrm{TM}} 31$ Oct 2011. doi: 10.1007/ s00262-011-1127-y

4. Gatault S, Legrand, F, Delbeke M, Loiseau S, Capron M (2012) Involvement of eosinophils in the anti-tumor response. Cancer Immunol Immunother. Online First ${ }^{\mathrm{TM}} 17$ June 2012. doi: 10.1007/ s00262-012-1288-3

5. Daniels TR, Martínez-Maza O, Penichet ML (2011) Animal models for IgE-meditated cancer immunotherapy. Cancer Immunol Immunother. Online First ${ }^{\mathrm{TM}} 22$ Dec 2011. doi: 10.1007/ s00262-011-1169-1

6. Karagiannis SN, Josephs DH, Karagiannis P, Gilbert AE, Saul L, Rudman SM, Dodev T, Koers A, Blower PJ, Corrigan C, Beavil AJ, Spicer JF, Nestle FO, Gould HJ (2011) Recombinant IgE antibodies for passive immunotherapy of solid tumours: from concept towards clinical application. Cancer Immunol Immunother. Online First ${ }^{\mathrm{TM}} 2$ Dec 2011. doi: 10.1007/s00262011-1162-8

7. Spillner E, Plum M, Blank S, Miehe M, Singer J, Braren I (2012) Recombinant IgE antibody engineering to target EGFR. Cancer Immunol Immunother. Online First ${ }^{\mathrm{TM}} 7$ June 2012. doi: 10.1007/s00262-012-1287-4

8. Castells M, Sancho-Serra MC, Simarro M (2012) Hypersensitivity to antineoplastic agents: mechanisms and treatment with rapid desensitization. Cancer Immunol Immunother. Online First $^{\mathrm{TM}} 11$ May 2012. doi: 10.1007/s00262-012-1273-X

9. Bluth MH (2011) IgE and chemotherapy. Cancer Immunol Immunother. Online First ${ }^{\mathrm{TM}} 2$ Dec 2011. doi: 10.1007/s00262011-1170-8

10. Mechtcheriakova D, Svoboda M, Meshcheryakova A, JensenJarolim E (2012) Activation-induced cytidine deaminase (AID) linking immunity, chronic inflammation and cancer. Cancer Immunol Immunother. Online First ${ }^{\mathrm{TM}} 23$ Apr 2012. doi: 10.1007/ s00262-012-1255-Z 